\title{
HACIA DESCENDENCIAS SALUDABLES: ALGUNOS ORÍGENES DEL DIAGNÓSTICO PRENATAL EN ESPAÑA
}

\author{
María Jesús Santesmases \\ Instituto de Filosofía, Centro de Ciencias Humanas y Sociales, CSIC
}

\section{RESUMEN}

Este trabajo reflexiona sobre las prácticas de diagnóstico prenatal en España. Con este fin se manejan tanto bibliografía sobre los orígenes de estas prácticas en otros países como datos encontrados en las primeras publicaciones al respecto de especialistas de nuestro país. Estas publicaciones se relacionan también con algunas previas sobre diagnóstico genético en la clínica en el caso del síndrome de Down. Se sugiere que las normas sociopolíticas propias de la dictadura de Franco se combinaron con la difusión de técnicas desarrolladas en el extranjero para estabilizar prácticas médicas asociadas a una reconceptualización del embarazo. Las técnicas de diagnóstico prenatal, pese a invisibilizar el cuerpo de las mujeres, mantienen a este en el centro de la tecnificación de las opciones reproductivas contemporáneas.

PALABRAS CLAVE: Cromosomas, genética, embarazo, diagnóstico prenatal, síndrome de Down.

\section{TOWARD HEALTHY OFFSPRINGS: EARLY PRENATAL TESTING IN SPAIN}

\section{ABSTRACT}

This paper deals with prenatal diagnosis practices in Spain. For pursuing this aim it reviews both literature on the origins of these practices in foreign countries as well as some of the early publications by Spanish practitioners. Those publications appeared to be connected to previous genetic testing in children such as the case of Down syndrome. Socio-political norms and values of Franco's regime together with clinicians' interests on introducing new testing techniques resulted in the stabilization of these practices associated to a reconceptualisation of pregnancy. Although prenatal diagnosis techniques made the body of pregnant women invisible, women's bodies remained at the core of the technicalisation of contemporary reproductive options.

KEY WORDS: Chromosomes, genetics, pregnancy, prenatal diagnosis, Down syndrome.

En medio de la creciente medicalización, tecnificación y diríase que también de la genetización del embarazo, los procedimientos de las sucesivas 
tecnologías médicas del siglo XX, desde los rayos $\mathrm{X}$ a finales del siglo XIX hasta los escáneres y tomografías más sofisticadas, han hecho los cuerpos cada vez más invisibles, como recuerda Tilly Tansey (2000) ${ }^{1}$. En el caso del embarazo, pese a esa invisibilidad técnica, los cuerpos de las mujeres permanecen como soportes de salud, malformación o enfermedad, de forma que los procedimientos del diagnóstico prenatal, aunque oculten ese continente físico para concentrarse en el feto, mantienen a las madres como portadoras. El feto como objeto de estudio aparecido en las imágenes diagnósticas médicas es accesible solo a través del cuerpo de la madre quien, además de acarrearlo literalmente en el abdomen, le transmite forma física y función biológica, a la vez que comparte con ese ser por nacer su propia opción reproductiva.

Los conceptos de salud y enfermedad son, por su parte, plásticos, flexibles, y las fronteras han resultado móviles - algunas enfermedades se convierten en desórdenes crónicos apenas perceptibles a simple vista por acción de fármacos, por ejemplo en algunos casos de diabetes; otras han encontrado modos de prevenir sus manifestaciones.

Este trabajo propone una aproximación al estudio de los orígenes de las prácticas de diagnóstico prenatal en España, que se relacionan con los primeros trabajos de genética humana en nuestro país. Las prácticas de diagnóstico prenatal estudiadas en este artículo se inician a partir de los diagnósticos de genética humana en los primeros años de la década de 1960. A su vez, se sugiere aquí una conexión entre estas y la formación de genetistas en España.

La clínica es el soporte institucional y cognitivo de las prácticas de genética humana estudiadas aquí, que se introducen a través de las especialidades de pediatría y de obstetricia conectadas a los análisis clínicos. A lo largo de ese proceso tiene lugar la aceptación de factores genéticos, citogenéticos en

1 TANSEY, T. (2000), «Introduction», en Looking at the unborn: Historical aspects of obstetrics ultrasound, A Witness Seminar held at the Wellcome Institute for the History of Medicine. En TAnsey, E. M., Christie, D.A. (eds.), Londres, The Wellcome Trust, p. i, http://catalogue.wellcome.ac.uk/record=b1473079a. Sobre el proceso de creciente medicalización del embarazo véase OAKLEY, A. (1984), The captured womb: A history of the medical care of pregnant women, Oxford-New York, Basil Blackell. En el caso de España, la autoridad médica como autoridad experta para una ciudadanía inmadura, también en el caso de las madres, durante el primer franquismo se trata en JiMÉNEZ LUCENA, I., RUIZ SOMAVILLA, M.J., CAStellanos Guerrero, J. (2002), «Un discurso sanitario para un proyecto político. La educación sanitaria en los medios de comunicación de masas durante el franquismo», Asclepio, 44, 201-218. Vase también AKRICH, M., PASVEER, B. (1996), Comment la naissance vient aux femmes: les techniques de l'accouchement en France et aux Pays-Bas, Le PlessisRobinson, Synthélabo Groupe. 
este caso, en las formas de pensar y practicar el diagnóstico, primero en niños y niñas y posteriormente se adopta al feto como objeto de estudio constituyéndose así la propia práctica del diagnóstico prenatal.

La estabilización en el uso de estas técnicas se produjo precisamente durante los años en los que resurgió el movimiento feminista; es decir, sintonizó con el derecho que las feministas reclamaban a decidir sobre su propio cuerpo y sobre si interrumpir o no el embarazo, en su caso. La dictadura de Franco había restringido en España el movimiento feminista y sus manifestaciones, como hizo con el asociacionismo y la actividad política en general ${ }^{2}$.

La muerte del dictador en 1975 y el restablecimiento de la democracia a partir de 1977 generaron expectativas, también en lo que respecta al derecho al aborto, al que se incorporó la indicación terapéutica. Una vez aprobada la ley de interrupción voluntaria del embarazo en 1985, el aborto continuó siendo, sin embargo, discutido y rebatido públicamente en foros sociales y profesionales $^{3}$. Y la indicación terapéutica ha sido esgrimida como indicación eugenésica para expresar actitudes contrarias al derecho de las mujeres a interrumpir un embarazo voluntariamente. Pero puede argumentarse también que la selección de los más dotados es un tema de discusión que forma parte de las preocupaciones de muchas mujeres y desde el pensamiento feminista se ha puesto en cuestión esta práctica ${ }^{4}$. El resultado es que el diagnóstico prenatal permite una selección, parcial, incompleta, siempre limitada y de cuyas limitaciones suele informase en muchos servicios de atención médica a las mujeres que aceptan someterse a ellas durante el embarazo. Lo que la información médica transmite a lo largo de las tres décadas de democracia en España es, como ha señalado Matiana González, una creciente genetización del discurso público y científico, que privilegia los factores genéticos en detrimento de otros 5 . Esto puede decirse del diagnóstico prenatal, que pudo haber seguido una trayectoria similar. De ahí el preguntarse por la procedencia de esa idea de que el diagnóstico prenatal pueda evitar el nacimiento de personas insanas, anormales y enfermas y el énfasis que se pone en ocasiones en el

2 SCANlON, G. M. (1986), La polémica feminista en la España contemporánea, $2^{\mathrm{a}}$ ed., Madrid, Akal.

3 BARreiro, B. (2000), Democracia y conflicto moral: la política del aborto en España e Italia, Madrid, Istmo.

4 Véase, entre otros, Lippman, A. (1994), «The Genetic Construction of Prenatal Testing: Choice, Consent, or Conformity for Women?». En RothenberG, K., Thomson, E. (eds.) Women and Prenatal Testing, Columbus, Ohio State University Press.

5 GonzÁlez Silva, M. (2005), «Del factor sociológico al factor genético: genes y enfermedad en las páginas de El País (1976-2002), Dynamis, 25, 487-512. 
hecho de que estas prácticas han disminuido el índice de nacidos con, por ejemplo, síndrome de Down ${ }^{6}$.

Inmersos en estas culturas de expansión del conocimiento genético se producen los debates previos a la aprobación en España, en 1985, de la Ley de interrupción voluntaria del embarazo y, en ella, de la indicación de aborto terapéutico en casos de riesgo de la salud del feto ${ }^{7}$. Este trabajo indaga en algunos aspectos de la construcción de esa práctica de aborto terapéutico por medio del rastreo de la introducción del diagnóstico genético y del diagnóstico prenatal. Las fuentes manejadas, esencialmente publicaciones médicas y clínicas junto a la historiografía disponible sobre casos extranjeros, muestran que el concepto de indicación terapéutica del aborto está directamente relacionado con los trabajos clínicos y médicos previos de diagnóstico de malformaciones y desórdenes en la salud infantil, trabajos que permitieron la estabilización y la posterior extensión de esas prácticas.

No pueden tratarse todos estos aspectos en este artículo, que no es más que un conjunto de reflexiones producido por resultados todavía preliminares de una investigación en curso. Aquí se revisan algunas publicaciones españolas sobre genética humana y diagnóstico prenatal aparecidas entre mediados de los años 1960 y mediados de los 1970 y se indaga en las posibles raíces de estas prácticas clínicas. Se trata por lo tanto de traer a colación los múltiples aspectos que participaron en su desarrollo, de modo que no resulte invisible que todos ellos intervienen como agentes en la construcción de la imagen pública del embarazo, de la percepción de las técnicas mismas y de los valores culturales que incorporan.

\section{Pensamiento hereditario}

La promoción del nacimiento de seres humanos (bien) dotados y saludables está originada por las prácticas eugenésicas desde la Inglaterra del siglo XIX en plena industrialización diseñadas por Francis Galton. Las propuestas para favorecer una reproducción que puede calificarse de adecuada se constituyó en proyecto social ligado a ideas de mejora de la especie humana (Álvarez 1985, 1988; Kevles 1985) ${ }^{8}$. Las prácticas nazis con los judíos para la purifica-

6 Véase por ejemplo «Los nacidos con anomalías congénitas son ahora la mitad que hace diez años», El País, 19 de agosto de 2004, p. 21.

7 BARREIRO (2000).

8 Álvarez, R. (1985), Sir Francis Galton, padre de la eugenesia, Madrid, CSIC. ÁL- 
ción de la raza aria pudieron desacreditar la eugenesia pero las nuevas prácticas experimentales y clínicas relacionadas con la biología celular tras la Segunda Guerra Mundial contribuyeron a reubicar el concepto de dotación genética, que se ligó a nuevos significados de salud y enfermedad asociados a tecnorituales diagnósticos. Algunos ejemplos se encuentran en las investigaciones sobre células fetales procedentes del líquido amniótico emprendidas en otros países; desde el primer momento se difundieron los análisis de células fetales procedentes del líquido amniótico como métodos para detectar posibles malformaciones ligadas al sexo en caso de mujeres pertenecientes a familias con esas enfermedades, como la hemofilia. Los primeros trabajos que describían métodos de determinación del sexo fetal, que se publicaron en 1955, se toman como origen de las técnicas de diagnóstico prenatal, pues uno de los grupos informó del aborto practicado a una mujer portadora de hemofilia tras identificarse su feto como de sexo masculino ${ }^{9}$.

Pero el proceso en España parece significativamente distinto, pues lo que se encuentra es una introducción inicial del diagnóstico genético que correlacionaba caracteres anatómicos y de salud con identificaciones cromosómicas, para, por lo visto hasta ahora, a continuación introducirse el diagnóstico antenatal, al abordarse el estudio del líquido amniótico procedente de mujeres embarazadas. La posibilidad de interrumpir el embarazo por riesgo en la salud del feto apenas se menciona o si se hace es de forma indirecta o implícita, o para dar razones por las que no se tiene en cuenta. Ello debe relacionarse con la prohibición del aborto en España en esos años, al mismo tiempo que pudo haberse contribuido a sentar las bases para estabilizar un conjunto de técnicas que contribuirían a la posterior aceptación de la indicación terapéutica en la interrupción voluntaria del embarazo en la democracia ${ }^{10}$.

Junto a las singularidades políticas, la introducción de estas técnicas en España comparte con el proceso que se lleva a cabo en otros países el hecho de generar un nuevo escenario para el diagnóstico de algunas de las características innatas en los fetos dentro del útero materno. Algunas malformacio-

VAREZ, R. (1988), Francis Galton. Herencia y eugenesia, Madrid, Alianza. KevLes, D. J. (1985), In the name of eugenics. Genetics and the uses of human heredity, Berkeley-Los Angeles, University of California Press.

9 SChWARTZ COWAN, R. (1994), «Women's role in the history of amniocentesis and chorionic villi sampling». En ROTHENBERG.,THOMPSON (eds.), pp. 35-47. Véase también Gaudillière, J. P. (2001), «Bettering Babies: Down's Syndrome, Heredity and Public Health in Post-war France and Britain». En Löwy, I, KRIGE, J., (eds.) (2001), Images of Disease. Science, Public Policy and Health in Post-war Europe, Luxemburg, Office for Official Publications of the European Communities.

10 BARREIRO (2000). 
nes y desórdenes considerados innatos y que se habían analizado con técnicas citogenéticas previamente en niñas y en niños se exploraron también al observarse que el líquido amniótico, que contenía células fetales, podía extraerse de forma segura y con riesgos pequeños de pérdida del embarazo ${ }^{11}$. De esta forma las redes de profesionales de la investigación experimental y clínica, en las que se incluyen aparatos y técnicas de laboratorio comenzaron a incluir cuerpos de mujer embarazada. Sin embargo, esas mismas prácticas, con el manejo de las muestras de células fetales extraídas del abdomen de las mujeres, han hecho del útero un continente invisible para las técnicas de cultivo celular y de diagnóstico fetal ${ }^{12}$. Al mismo tiempo y pese a esa transparencia del cuerpo de la madre, es ésta la portadora de salud y enfermedad del feto que acarrea ${ }^{13}$. Así alcanza el feto un protagonismo notable como objeto de estudio clínico a la vez deviene parte de un proceso de práctica cultural, social, de una eugenesia nueva. La salud de la madre, y en muchos casos también la del padre, se correlaciona con el análisis de la de su feto para explicar y razonar sobre la introducción de los diagnósticos antenatales - así se llamaron inicialmente- en la práctica clínica del seguimiento del embarazo.

\section{Pensamiento HeREDITARIO Y DEFECTOS CONGÉNITOS}

Entre las prácticas diagnósticas prenatales y el pensamiento sobre la herencia biológica puede establecerse una cierta continuidad. Estas técnicas evocan trabajos previos de genética hoy denominada clásica: estudios de poblaciones, y trabajos procedentes de la transmisión de caracteres hereditarios en plantas y animales que hunden sus raíces en prácticas antiguas de cría se-

11 Una historia breve del diagnóstico prenatal está en PINELL, P. (2004), «Diagnostique prénatal». En LeCOURT, D. (dir.), Diccionnaire de la pensée médicale, París, Quadrige-PUF, pp. 333-337. Véase también Schwartz Cowan, R. (2001), «Medicine, Technology and Gender in the History of Prenatal Diagnosis». En Creager, A., LunBeCK, E.y SCHIEBINGer. L. (eds.) (2001), Feminism in Twentieth-century Science, Technology and Medicine, Chicago, Chicago University Press. SchwARTZ CowAN, R. (1994) y GAUDILLIÈRE (2001).

12 Sobre la imagen pública del feto como ente autónomo ajeno al útero, véase GILBERT, S. F., HowES-Mischel, R. (2004), «'Show me your original face before you were born': the convergence of public fetuses and sacred DNA», History and Philosophy of the Life Sciences 26, 377-394.

13 Sobre la visibilidad del feto y la transparencia del cuerpo de la madre, véase también el estudio de Lisa Mitchell sobre la ecografía: MitcheLl, L. M. (2001), Baby's first picture. Ultrasound and the politics of fetal subjects, Toronto-London, University of Toronto Press. 
lectiva en la jardinería, la agricultura y en la ganadería ${ }^{14}$. Pero el diagnóstico prenatal incluye un conjunto de técnicas e instrumentos cuyos orígenes son los procedimientos de laboratorio puestos a punto desde el periodo de entreguerras, inicialmente en estudios de cromosomas y manejo de técnicas citológicas, de cultivos de células para su estudio. La forma que toman los procedimientos de cultivo celular después de la Segunda Guerra Mundial son las que marcan diferencias respecto a técnicas y conceptos previos; no son meramente técnicos sino que devienen prácticas clínicas y culturales que arraigaron en la imagen del embarazo y de nacimientos saludables que son hoy parte del pensamiento biológico y social ${ }^{15}$.

El interés por los defectos congénitos, en cuyo estudio está basado precisamente el concepto de herencia biológica, fue una de las bases de lo que hoy se denomina genética, relacionado con el carácter heredable de algunas características calificadas de monstruosas y en el estudio de linajes. Ideas y conceptualizaciones de naturalistas y médicos que negociaban espacios de movilización profesionales fraguaron la dicotomía naturaleza/medio a través de los cuales, como ha analizado Carlos López Beltrán, se estabilizó el concepto naturaleza - ligado a la herencia biológica - y se desplazó la importancia del segundo, medio o cultura, como mera «comparsa del motor hereditario» ${ }^{16}$.

La preocupación y la sensibilidad social hacia el asunto de la salud de los embriones o fetos a partir de finales de la década de 1950, en el caso de mujeres embarazadas, podrían estar relacionados, por un lado, con los efectos congénitos de las radiaciones. Desde los trabajos de H.M. Muller previos a la Segunda Guerra Mundial sobre los efectos de los rayos X en Drosophila hasta los estudios de las poblaciones supervivientes en Hiroshima y Nagasaki en plena posguerra, se divulgó por todo el mundo el problema de la transmisión de malformaciones por alteraciones congénitas ${ }^{17}$.

Por otro lado, poco menos de una década después, las malformaciones con las que durante los años 1960 nacieron niños y niñas en Europa de mujeres

14 Esas raíces están también en los orígenes de las prácticas de Mendel. Para un caso de la agricultura, sobre la cría de ganado, vease RUSELL, N. (1986), Like engend'ring like: Heredity and animal breeding in early modern England, Cambdrige, Cambridge University Press. Sobre Mendel y su influencia, el ya clásico de OLBY, R. (1985), Origins of mendelism, Chicago: University of Chicago Press, entre otros muchos.

15 SCHWARTZ COWAN (2001).

16 Véase López Beltrán, C. (2004), El sesgo hereditario, México D.F., Universidad Nacional Autónoma de México.

17 Sobre los estudios a supervivientes de Hiroshima, véase LINDEE, S. (1994), Suffering made real. American science and the survivors at Hiroshima, Chicago, University of Chicago Press. 
que habían sido tratadas con talidomida para evitar los mareos y malestares habituales, contribuyeron, entre otras cosas a extender la preocupación ${ }^{18}$. El desarrollo de las técnicas de extracción y análisis de líquido amniótico, la ecografía y otras técnicas posteriores de diagnóstico antenatal pudieron contribuir a atender esas inquietudes.

\section{EMBARAZO Y GENÉTICA HUMANA}

«[L]a comprobada transmisión hereditaria de multitud de defectos han de ser los fundamentos de una eugenesia prudente, sin llegar a extremos incompatibles con los derechos y la dignidad humanas» (Ramón Margalef, 1953) ${ }^{19}$.

En su breve recuento de la historia del diagnóstico prenatal en Francia, Patrice Pinell (2004) se refiere, como hace Gaudillière (2002), a una nueva eugenesia. Se contrapone así este concepto como novedad respecto a una vieja eugenesia, que contribuyó a las raíces del nazismo ${ }^{20}$. Datos recientes ilustran la influencia que las técnicas de diagnóstico prenatal han tenido en los datos estadísticos de defectos al nacimiento, por un lado, y en el pensamiento social y público sobre descendencia saludable, por otro ${ }^{21}$.

Ello ha conducido a la construcción del concepto de embarazo saludable en el medio cultural que se ha desarrollado desde Segunda Guerra Mundial. Si en los inicios de la promoción de la genética el embarazo no fue centro de atención de la experimentación, tras la guerra el pensamiento hereditario y el desarrollo de algunas técnicas particulares, como la asistencia clínica de embarazos complicados, propiciaron cruces interdisciplinares entre genetistas procedentes de la biología y de la agricultura y la ganadería, y clínicos de

18 Para un repaso del caso de la talidomida y sus nuevos usos, véase TimmERmans, S., LEITER, V. (2000), «The redemption of thalidomide: standarizing the risk of birth defects», Social Studies of Science 30, 41-71.

19 Margalef, R (1953), La herencia biológica, Barcelona, Seix Barral, p. 161.

20 Sobre eugenesia, véanse KEVLES (1985) y ÁLVAREZ (1985) y (1988).

21 Una organización internacional creada en 1974, la International Clearing House for Birth Defects, recoge información proporcionada con carácter voluntario sobre defectos al nacimiento y sobre programas de investigación y prevención de estos. Obtiene datos de forma sistemática de una treintena de países y está asociada a la Organización Mundial de la Salud. Véase http://www.icbdsr. org/page.asp? $\mathrm{n}=$ AboutUs (la consulta más reciente es de 20 de septiembre de 2007). Véase también la segunda edición del World Atlas of Birth Defects en http://www.who.int/genomics/ about/en/1-4.pdf (consultado por última vez el 20 de septiembre de 2007). 
diversas especialidades; obstetricia y ginecología es solo una de ellas, pues también intervinieron la pediatría y los departamentos de análisis clínicos ${ }^{22}$.

Todo ello coincide con intereses de algunos clínicos españoles de incluir en la práctica médica los entonces nuevos métodos científicos y técnicos disponibles, terapéuticos y diagnósticos, en su difusión inicial. Los procedimientos de adquisición de técnicas y de regulación de su uso parecen en sintonía con los de introducción de nuevos fármacos tras el fin de la segunda guerra mundial. En los casos de adopción de terapias con antibióticos y hormonas — entre ellas la insulina-, el laboratorio y la clínica establecen contactos, cruces, interacciones: intercambian prácticas y pensamiento médico y biológico. No es que la clínica acudiera al laboratorio sino que incorpora este a sus prácticas médicas y de atención sanitaria. Y mientras tanto, la autoridad médica y clínica ejerce una función reguladora de las nuevas prácticas diagnósticas y terapéuticas.

En ese marco se reciben en España los resultados de los análisis citogenéticos en la década de 1960. Los servicios de pediatría de algunos grandes hospitales diagnosticaban por caracteres anatómicos algunas malformaciones que comenzaron a calificarse de genéticas al poder diagnosticarse y definirse también por medio de análisis citológicos en los cuales los cromosomas podían contarse.

\section{LA FORMACIÓN DE GENETISTAS EN ESPAÑA}

Las investigaciones en genética en España comenzaron y se desarrollaron con éxito durante el primer tercio del siglo $\mathrm{xx}$, cuando hubo investigaciones al día en lo que fue durante ese tiempo la fragua de la genética experimental de plantas y veterinaria ${ }^{23}$. Por lo que respecta a la docencia universitaria tras la Guerra Civil, en 1953 se estableció la asignatura de Genética en los estudios universitarios de Ciencias, en la rama de Biología y Geología y también, unida a la mejora de plantas, en la Escuela de Ingenieros Agrónomos. En la Escuela de Agrónomos de Madrid, Vicente Boceta, discípulo del genetista Cruz

22 Para una historia de la genética de plantas y animales, no humana, en España, véase PINAR, S. (2002), «La vertiente histológica de José Fernández Nonídez, introducción de la teoría mendeliano-cromosómica en España», Asclepio, 54, 3-18; PINAR, S. (2002), «The emergence of modern genetics in Spain and the effects of the Spanish civil war», Journal of the History of Biology 35: 111-148; así como los textos recogidos en CANDELA, M. (ed.)(2003), Los orígenes de la genética en España, Madrid, Sociedad Estatal de Conmemoraciones Culturales, entre ellos el de PINAR, S. (2003), «La genética española en la primera mitad del siglo XX», en pp. 15-72.

23 Sobre las investigaciones en genética en España durante ese periodo véase PINAR (2002) y (2003). 
Gallástegui, obtuvo el puesto de profesor por concurso y cuando murió, en 1957, fue sustituido por Enrique Sánchez-Monge, quien obtuvo la cátedra de esa escuela por oposición en 1960. En la Facultad de Ciencias de Madrid Antonio de Zulueta fue el primer profesor de genética seguido de Eugenio Ortiz; en la de Barcelona estuvo impartida por Antonio Prevosti. Las primeras cátedras que se dotaron en las facultades de Ciencias de Madrid, Barcelona y Granada, con ese nombre en 1963 fueron cubiertas por oposición por Enrique Sánchez-Monge, Antonio Prevosti y Eugenio Ortiz, respectivamente. Todos ellos realizaron investigaciones durante sus años docentes ${ }^{24}$.

De la importancia de la genética en la cría de animales es reflejo la existencia de la asignatura Zootecnia en los estudios de veterinaria, que incluía estudios de genética así como de alimentación y que se desdobló más tarde, para crearse un Departamento de Genética en la Facultad de Veterinaria de Madrid.

De los primeros docentes y catedráticos de genética españoles que desarrollaron su carrera académica durante el franquismo, Sánchez-Monge es al que por su trabajo investigador, y probablemente por haber sido profesor simultáneamente en dos centros universitarios durante varios años - la Escuela de Agrónomos y la Facultad de Ciencias madrileñas-, se recuerda como uno de los más productivos e influyentes. Publicó libros y manuales de genética, y glosarios de términos mientras desarrollaba sus investigaciones sobre mejora genética de plantas y cultivos, estudios y libros que obtuvieron un amplio reconocimiento en España ${ }^{25}$. Suele recordársele también por haber formado a especialistas que, procedentes de los estudios universitarios de biología, se convertirían en genetistas y que desarrollarían sus trabajos en la clínica ya que la genética humana, desarrollada en departamentos hospitalarios desde al menos la primera mitad de la década de 1960, no contó con estudios en la licenciatura de medicina en España ${ }^{26}$. Su primera etapa como investigador en la Esta-

24 Véase CANDELA, M. (2003), «Los primeros genetistas españoles en la segunda mitad del siglo XX». En Candela, M. (ed.)(2002), Los orígenes de la genética en España, Madrid, Sociedad Estatal de Conmemoraciones Culturales, pp. 72-112.

25 Sobre Sánchez-Monge, véase Jouve DE LA BARredA, N. (2003), «Don Enrique Sánchez-Monge y Parellada, impulsor de la mejora genética de plantas», en CANDELA, M. (ed.), Los orígenes de la genética en España, Madrid, Sociedad Estatal de Conmemoraciones Culturales, pp. 397-422.

26 Una muestra del respeto académico hacia Sánchez-Monge es su elección en 1968 como académico de número de la Real Academia de Ciencias Exactas, Física y Naturales, y la contestación a su discurso de ingreso por el biólogo Florencio Bustinza, que menciona muchos detalles de la producción científica y de manuales para la docencia de la genética de Sánchez-Monge. Véase SÁNCHEZ-Monge, E. (1971), La androesterilidad vegetal y su utiliza- 
ción Experimental de Aula Dei del Consejo Superior de Investigaciones Científicas en Zaragoza dedicado a la mejora de especies vegetales y agrícolas coincidió con la estancia en ese centro de Joe Hin Tjio, uno de que estuvo entre los pioneros en estudios de cromosomas humanos, como se verá más adelante.

\section{INTRODUCCIÓN DE ANÁLISIS CITOGENÉTICAS EN ESPAÑA: LA CLÍNICA}

Uno de los inicios de las técnicas puestas a punto con fines de diagnóstico genético en España procede del interés del médico Carlos Jiménez Díaz por contar con una clínica actualizada y capaz de incorporar nuevas técnicas experimentales al trabajo médico. Bajo su dirección, la Clínica de la Concepción de la Fundación Jiménez Díaz en Madrid, creada y dirigida por él mismo, se constituyó en promotora de la formación en medicina experimental y en investigación médica ${ }^{27}$.

En la Fundación Jiménez Díaz, el cardiólogo Andrés Sánchez Cascos fue quien organizó el servicio de diagnóstico genético. Doctorado en cardiología, estudió los aspectos genéticos de las cardiopatías. Tras una estancia en el Guy's Hospital de Londres, a su regreso puso en marcha el servicio de diagnóstico genético que se convertiría en una Unidad de Genética ${ }^{28}$. Sánchez Cascos había presentado su tesis para el grado de doctor en Medicina sobre «Correlación electrodinámica de las cardiopatías congénitas» en 1960 en la Universidad de Madrid ${ }^{29}$. Es decir, trabajó en el carácter congénito de las enfermedades del corazón.

ción. Discurso leído en el acto de su recepción y contestación de Florencio Bustinza, Madrid, Academia de Ciencias Exactas, Físicas y Naturales.

27 Sobre Jiménez Díaz, veáse la reconstrucción sobre sus propios intereses investigadores y clínicos en JiMÉNEZ DíAZ, C. (1965), La historia de mi instituto, Madrid, Paz Montalvo. Una biografía informativa y apasionada está en JiMÉnEz CASADO, M.(1993), Doctor Jiménez Díaz: vida y obra, Madrid, Fundación Conchita Rábago de Jiménez Díaz.

28 En el Guy's Hospital de Londres el médico Paul E. Polani había investigado sobre las causas de las enfermedades cardíacas congénitas y sobre intersexos y trabajó en colaboración con Charles Ford, que investigaba también en las técnicas de identificación de cariotipos en Inglaterra. Véase KEVLES (1985), pp.. 242-244. He podido saber del papel de Sánchez Cascos en varias entrevistas a genetistas españolas: entrevista a María Jesús Lautre, 14 de septiembre de 2001; entrevista a María Luisa Martínez Frías, 13 de septiembre de 2004; entrevista con Carmen Ramos, 2 de noviembre de 2004.

29 Tesis inédita, Facultad de Medicina, Universidad de Madrid 1960. Posteriormente, en 1970, publicaría Etiología de las cardiopatías congénitas, Madrid, Paz Montalvo. 
Al regreso, al menos desde 1962, había en la Fundación Jiménez Díaz un servicio de diagnóstico citogenético dirigido por Sánchez Cascos al día en las técnicas que empezaban a utilizarse para realizar diagnósticos citogenéticas. Este tipo de diagnósticos tenía por objeto en ese momento la identificación y caracterización de los cromosomas humanos por medio de técnicas de cultivo celular que permitían observarlos al microscopio sin que solaparan unos con otros en la muestra. La identificación cromosómica con una malformación correlacionaba así a esta con la genética, la dotó de carácter congénito, heredable.

El procedimiento para el recuento de cromosomas había sido puesto a punto por Joe Hin Tjio, que colaboraba de modo permanente en la Estación Aula Dei de Zaragoza (España), y Albert Levan, del Instituto de Genética de Lund (Suecia), en investigaciones conjuntas en tejidos humanos ${ }^{30}$. Basado en su pericia como citólogos de plantas que se dedicaron después a células animales, el método era una aplicación del desarrollado por T.C. Hsu para otro tipo de células: nuevas técnicas de digestión de las muestras les permitieron obtener cromosomas separados de tal modo que tras su cultivo era posible observarlos con claridad al microscopio y clasificarlos. El trabajo conjunto de Tjio y Levan en Lund (Suecia) en el año 1955, incluyó la búsqueda de cromosomas en células de pulmón fetal procedentes de abortos legales. Fue en ellas donde contaron 46 cromosomas en vez los 48 que se tenían por el número de cromosomas en las células humanas. El resultado se reproducía y finalmente lo publicaron en 1956. Fue posteriormente confirmado por Charles Ford y John Hammerton en Inglaterra ${ }^{31}$.

30 Joe Hin Tjio consta como colaborador permanente de esta Estación en las memorias del CSIC de 1949 con la «señora de Tjio» (p. 265). En las memorias correspondientes a los años 1952-1954 figura entre el «Personal directivo» como «Profesor extranjero» e «Ing. Agr.» (se entiende que ingeniero agrónomo) (págs. 757 y 759 respectivamente), que colaboraba en «Estudio de cromosomas animales» con Levan, del Instituto de Genética de Lund y del Laboratorio del Cáncer en Lund (Suecia). Los estudios de Tjio en Aula Dei prosiguieron al menos hasta 1958, según las memorias del CSIC de 1955-57 y 1958. Véase http://www.csic.es/memorias. En 1960 figura ya en los National Institutes of Health en Bethesda (Maryland, Estados Unidos), según la lista de participantes en la reunión de Denver (Colorado) de ese año para establecer estándares en la nomenclatura de los cromosomas humanos: véase «Proposed standard system of nomenclature of human mitotic chromosomes», The Lancet 1 (1960), 1063-1065, en la p. 1065. Como adscrito a Aula Dei figura en la primera publicación con Levan sobre el número de cromosomas humanos en la revista publicada en Lund por la Asociación Escandinava de Genetistas: TJIO, J. H., LEVAN, A. (1956), «The chromosome number of man», Hereditas 42, 1-6

31 Los experimentos por los cuales Tjio y Levan establecieron en 1956 que el número de cromosomas humano era 46, y no 48 como hasta entonces se creía, fue posible por el uso de técnicas citogenéticas descritas a principios de la década de 1950. Véase HSU, (1979). 
Uno de los primeros desórdenes en estudiarse por técnicas citogenéticas fue el síndrome de Down. Había sido descrito a mediados del siglo XIX por J. Langdon Down asociado a deficiencia metal y a caracteres anatómicos concretos. En la década de 1950 se estableció la correlación entre esos caracteres anatómicos y el retraso mental con los cromosomas y así con las prácticas citogenéticas. Fue en 1959 cuando Jérôme Lejeune, Marthe Gauthier y Raymond Turpin en el Hospital Trousseau de París describieron que sus pacientes con síndrome de Down poseían 47 cromosomas en vez los 46 que Tjio y Levan habían descrito para los cromosomas humanos en $1956^{32}$.

En 1964, Sánchez Cascos y colaboradores en Madrid describieron veinte casos de síndrome de Down en un estudio citogenético. Las muestras analizadas procedían de pacientes de la propia Clínica de la Concepción (Fundación Jiménez Díaz) y del Hospital Clínico, del Hospital de Niño Jesús, del Hospital Militar y del Hospital de la Cruz Roja. En ese trabajo se señala que ese diagnóstico tenía «utilidad en la práctica por la posibilidad de existencia de portadores sanos entre los familiares de los casos con algún tipo de mongolismo» así como «la futura profilaxis de más de un caso» ${ }^{33}$. En él se confirma para los casos de síndrome de Down la fórmula cromosómica de 47 cromosomas, con trisomía en el par 21. Esto sugiere que, si bien pudo carecerse de fines de diagnóstico prenatal de este tipo de análisis citogenéticos, se podría haber tenido información sobre su uso y las técnicas manejadas con éxito en diagnóstico antenatal, de análisis procedentes de muestras con células fetales, y su aplicación a lo que se denomina en ese trabajo como «profilaxis» y que solo podía consistir en evitar un embarazo o en interrumpirlo. Aunque es razonable pensar que los clínicos españoles tuvieran acceso a la información médica y a las publicaciones disponibles sobre ello, la promoción de la natalidad, política explícita durante el franquismo, tanto como la ilegalidad del aborto y las ideas respecto a esta práctica durante toda la dictadura (1939-1975) son agentes que participaron en la construcción de los intereses y los objetivos médicos y clínicos de este tipo de pruebas en aquel momento en España, como también lo fueron las informaciones y publicaciones médicas y biológicas que manejaban sobre novedades diagnósticas ${ }^{34}$.

32 Sobre Lejeune, véase KEVLES (1985), p. 247 y GAUDILLIÈRE (2001).

33 Sánchez Cascos, A., Morales, A. y Barreiro, E. (1964), «Estudio genético de veinte casos de síndrome de Down (mongolismo)», Revista Clínica Española XCIII (1), 2326, en la p. 23. Aparentemente los hospitales de los que procedían las muestras estaban todos en Madrid, su nombre corresponde a centros asistenciales con ese nombre, pero no se especifica en el texto citado.

34 Sobre la promoción de la natalidad en España, véase NASH, M. (1996), «Pronatalismo y maternidad en la España franquista». En G. BocK y P. THANE (eds.), Maternidad y políticas 
Mientras se empezaban a practicar estas nuevas técnicas citogenéticas, se mantenía al mismo tiempo el valor del diagnóstico del síndrome de Down basado en la uniformidad fisionómica. Sobre la importancia atribuida al diagnóstico fisionómico por los pediatras en la década de 1960 en España, son ilustrativas las afirmaciones del pediatra López Linares, de la Fundación Jiménez Díaz, quien afirmó en 1967 que «el mongolismo» podía diagnosticarse «con una sola mirada» y razonaba que «el diagnóstico realizado por un clínico experto es superior, en la mayoría de los casos, al estudio citogenético» ${ }^{35}$. Lo cual sugiere debates entre genetistas y pediatras al menos en aquel preciso momento, a mediados de la década de 1960, cuando comenzaban a impartirse los cursos de genética humana en la Fundación Jiménez Díaz ${ }^{36}$.

Desde el departamento de Citología de la Fundación Jiménez Díaz se siguió el proceso de identificación de anomalías cromosómicas, basadas en análisis citológicos. Estas eran en 1967 un número «casi inagotable». Hasta ese año y desde la creación en 1962 del Departamento de Genética de la Fundación Jiménez Díaz se había hecho el cariotipo a 500 personas; la mayoría portadoras de enfermedades congénitas pero también a familiares de ellas ${ }^{37}$.

En cuanto a las técnicas, Sánchez Cascos dio detalles sobre estas en el curso de genética humana cuyos contenidos se publicaron en 1967. La «más rápida, cómoda y barata», según su texto, usaba cultivos de leucocitos de sangre periférica. La receta del procedimiento se detalla sugiriendo que procedían de muestras de personas, ya nacidas, sin que se encuentre en este caso referencia alguna a células fetales. Una vez tratadas de acuerdo al procedi-

\footnotetext{
de género, Madrid, Cátedra-Instituto de la Mujer, pp. 279-307; y SCANLON, G. M. (1986), La polémica feminista en la España contemporánea, $2^{\mathrm{a}}$ ed., Madrid, Akal. Todos los trabajos consultados y citados aquí cuentan con numerosas citas de artículos científico-médicos extranjeros; pueden verse, por ejemplo, las de SÁNCHEZ CASCOS 1966, Etiopatogenia, pp. 121-123; y las referencias en LAUTRE, M. J. (1976), «Predicción antenatal de las anomalías cromosómicas». En Botella, J.y IzQuierdo, L. (eds.), Problemas actuales de genética humana, Madrid, Instituto de España, pp. 177-191.

35 López Linares, M. (1967), «Síndrome de Down». En SÁnchez Cascos, A., BarreiRo Miranda, E. (1967), Curso de genética humana, «con la participación de los Profs. J.Botella Llusiá, E. López García y J. Perianes Carro» [y diez autores más], Madrid, Fundación Jiménez Díaz, pp. 83-96; en pp. 92-93.

36 Sobre los contenidos de los primeros cursos véase SÁNCHEZ CASCOS y BARREIRO MIRANDA (1967) y SÁNCHEZ CASCOS, SAN ROMÁN y LAUTRE (1969).

37 Esos datos están recogidos en SÁnchez CASCOS, A, BARREIRO, E. (1967), «Apéndice: Estudio citogenética de 500 enfermos». En SÁnchez CASCos, A., BARREIRo MirandA, E. y otros (1967), Curso de genética humana, Madrid, Fundación Jiménez Díaz, pp. 201-sin numerar.
} 
miento técnico que describe, en el que se incluyen tinciones y análisis al microscopio así como realización de microfotografías, «se recortan los cromosomas y se pegan en cartulinas» para su clasificación y para la descripción del «mapa cromosómico», también denominado «genotipo». Con estas prácticas de reconstrucción del cariotipo, Sánchez Cascos seguía las recomendaciones sobre nomenclatura de cromosomas humanos que se había difundido tras la Convención de Denver. Esa reunión de diecisiete genetistas en Denver (Colorado, Estados Unidos) se había celebrado en 1960 a sugerencia del citogenetista británico de Harwell Charles Ford, con el fin de aunar criterios para la identificación y la clasificación de cromosomas, cuando estaba empezando a discreparse entre los distintos grupos que investigaban sobre ellos, con el fin de evitar, o minimizar, las cuales se pretendió crear referencias estables para la descripción de los cromosomas humanos ${ }^{38}$.

Sánchez Cascos menciona en ese mismo capítulo que la determinación de cromosomas obtenidos del líquido amniótico de mujeres embarazadas, extraído por punción, podía establecer «con exactitud» el sexo, se entendía que del feto; pero añadía que «dicha punción puede terminar en aborto, no siendo por tanto una técnica recomendable». Finalmente, termina con la conclusión de que «la gran aplicación de la Populogenética humana» era resolver «el angustioso problema del matrimonio que acaba de tener un hijo malformado o mongoloide y que nos pregunta si tendrá más hijos afectos» ${ }^{39}$. El texto sugiere, por lo tanto, que se hacían análisis citogenéticas a niños y niñas de poca edad, y que los resultados se relacionaban con la descendencia futura. La citogenética o, como él la denomina, la populogenética, comenzaba a incluir relaciones entre rasgos de salud y enfermedad, por un lado, y caracterización cromosómica, por otro, y a correlacionarla con la conveniencia o no de tener más descendencia.

Sánchez Cascos fue uno de los primeros especialistas españoles en genética que se introdujo en esta área desde la clínica humana, y llegó a ella desde la cardiología ${ }^{40}$. Tuvo un papel influyente en la extensión de las prácticas de

38 «A Proposed Standard System of Nomenclature of Human Mitotic Chromosomes, Denver, Colorado, 1960». En Birth Defects, Original Article Series. Chicago Conference: Standardization in Human Cytogenetics, New York, The National Foundation-March of Dimes, 1966.

39 Sánchez Cascos, A. (1967), «Cito y Populogenética Humanas». En SÁnChez CaSCOS, A., BARREIRo MirandA, E. y otros, (1967), pp. 5-14.

40 Véanse sus trabajos entre 1961 y 1964, todos ellos citados en SÁnCHEz CASCOS, A. (1970), Etiopatología de las cardiopatías congénitas, Madrid, Paz Montalvo. 
diagnóstico genético en España y desempeñó temporalmente la docencia de esa disciplina en la Facultad de Medicina, además de escribir manuales y coordinar publicaciones sobre esta disciplina entonces emergente.

\section{LA EXTRACCIÓN DE LÍQUIDO AMNIÓTICO}

Por su parte, el desarrollo de las técnicas de cultivo de células fetales permitió desde mediados de la década de 1960 establecer métodos reproducibles para obtener muestras celulares cuyo cultivo en medio adecuados permitiera también que los cromosomas aparecieran separados en el medio y fuera posible contarlos y observar sus formas ${ }^{41}$. Los primeros análisis de células fetales se referían al sexo. La identificación del sexo celular había sido descrita en 1949 por Murray L. Barr, citólogo de la Universidad de Western Ontario (Canadá) cuyas técnicas de tinción revelaron un pequeño satélite que aparecía en las células femeninas y no en las masculinas. Estos denominados «cuerpos de Barr», también llamados «cromatina sexual» se usaron para determinar el sexo y combinados con la observación de células fetales en el líquido amniótico permitieron la determinación prenatal del sexo ${ }^{42}$.

Los primeros análisis del líquido amniótico extraído del abdomen de mujeres embarazadas con fines diagnósticos se hicieron a principios de la década de 1950 para estudiar posibles incompatibilidades en el Rh y en casos de exceso de líquido amniótico. Del estudio de las células fetales podía concluirse el sexo del feto en función de la presencia o ausencia de cromatina sexual en las células fetales procedentes del cultivo de muestras de líquido amniótico. Por esa técnica se pudieron diagnosticar enfermedades ligadas al sexo, entre ellas la hemofilia y una distrofia muscular, aunque no de forma directa sino al relacionarse con la determinación del sexo del feto. En 1960 se empleó por primera

41 Kottler, M. J. (1974), «From 48 to 46: Cytological technique, preconception and the counting of human chromosomes», Bulletin of the History of Medicine 48, 465-502; HSU, T. C. (1979), Human and Mammalian Cytogenetics. An historical perspective, Nueva York, Springer-Verlag; MARTIN, A. (2004), «Can't any body count? Counting as an epistemic theme in the history of human chromosomes», Social Studies of Science, 34: 923-948.

42 Sobre el sexo y los cromosomas, véase Delgado Echeverría, I. (2007), El descubrimiento de los cromosomas sexuales, Madrid, CSIC. Sobre Barr, KEVLES (1985) p.242 y SCHWARTZ CowAN (1994). Nótese que la cromatina sexual de Barr no eran los cromosomas sexuales $\mathrm{X}$ e Y. 
vez para, una vez predicho el sexo masculino del feto de una embarazada que transmitía la hemofilia, practicarse el aborto tras el resultado positivo ${ }^{43}$.

Así pues las primeras informaciones extraídas del cultivo de células obtenidas por amniocentesis se usaron para determinar el sexo, basado en estudios previos sobre relación entre sexo y la transmisión de algunos caracteres anatómicos y patologías y síndromes observables en el ejercicio clínico. Los datos experimentales pioneros en este tipo de correlaciones se habían realizado en diferentes organismos por diversos biólogos y biólogas, entre los que suele destacarse el grupo de Thomas H. Morgan en Estados Unidos y sus investigaciones sobre la genética de la mosca de la fruta Drosophila melanogaster. De ese grupo proceden algunas de las más tempranas técnicas de cultivo de células para el estudio de los cromosomas. Las características limitadas por el sexo (hoy denominadas «ligadas al sexo») fueron uno de los primeros temas de investigación genética ${ }^{44}$.

Por esta vía pudieron llegar a identificarse el número de cromosomas y el exceso de éstos en el par 21, establecido por Lejeune, Gauthier y Turpin en Francia y que estabilizó esta caracterización citogenética del síndrome de Down, como ya se ha dicho. Ello llevó a practicar el aborto tras diagnósticos positivos de esta anomalía en Inglaterra. La posible generalización y la estandarización de esta práctica diagnóstica en los años siguientes fue objeto de discusiones entre genetistas y médicos. La edad de la mujer embarazada se convirtió en el criterio principal para recomendar la amniocentesis y el análisis de cromosomas. Durante los años 1970 se llegó a hablar de una posible eliminación del síndrome de Down entre la población por este método ${ }^{45}$.

Las amniocentesis, realizadas por indicación médica, empezaron a practicarse en España a principios de la década de 1970 y para detección del Rh del feto cuando se preveían complicaciones. Entre las primeras mujeres atendidas con esta técnica se encontraban aquellas que tenían exceso de líquido amniótico, por lo que se trataba de punciones evacuatorias. En 1975, Lautre, que se había formado en la Unidad de Genética de la Fundación Jiménez Díaz con Sánchez Cascos y en el Guy's Hospital de Londres, presentó un conjunto de

43 Sobre las informaciones y las prácticas en España en la década de 1970, véase LAUTRE (1976). Sobre otros países, Pinell (2002); GAUDILLIÈRE (2002) y KEVLES (1985).

44 Véase HubBard, R. (1997), The politics of women's biology, New Brunswick (New Jersey), Rutgers University Press, 1997.

45 Sobre los debates, KeVLes (1985), pp. 286-287. Sobre los orígenes de la amniocentesis y la detección prenatal de síndromes, véanse los trabajos ya citados de SCHWARTZ CowAN (1994) y también GAUDILLIÈRE (2001). 
resultados de estas prácticas. Explicó en esa ocasión que la amniocentesis se practicaba «por vía transabdominal bajo control ecográfico», lo que minimizaba el riesgo de daños al feto y a la madre, o por vía vaginal si no había localización previa del saco amniótico. Consistía en la extracción de 10 centímetros cúbicos, que se cultivaban con suero fetal. La duración del cultivo hasta obtener células adecuadas variaba. Pero, añadió Lautre, esa premura para obtener resultados no era importante en España, pues el aborto terapéutico no estaba autorizado por la ley. De los 67 casos presentados en aquella primera ocasión, solo dos presentaron alteración cromosómica. La ciencia estaba haciendo posible «el control genético del feto», según Lautre, pero añadía, «la prevención total de las anomalías genéticas llevaría a practicar el control prenatal en todos los embarazos», lo que resultaba «imposible», aunque podían establecerse criterios de riesgo, por edad e historia familiar. Finalmente concluyó afirmando que no pretendía «entrar en discusión acerca de lo ético, moral o permisible del aborto terapéutico para poner fin a los embarazos» en los que se había detectado anomalías y, afirmaba, «es misión del médico, concretamente del genetista, el informar del riesgo existente» ${ }^{46}$.

Mientras tanto, la introducción de la ecografía, para el estudio del feto en el interior del útero, reconfiguró el embarazo y el crecimiento del feto en el interior del abdomen de las mujeres embarazadas como un proceso observable in vivo y en directo por medio de los ultrasonidos ${ }^{47}$. La técnica se desarrolló a partir de mediados de la década de 1950 y su uso en la clínica del embarazo se estabilizó a partir de la década de 1970. Así fue como tras establecerse una técnica que identificaba el genotipo (la dotación cromosómica), se inventó y empezó a extenderse el uso de otra que regresaba a la observación del fenotipo, a la forma del cuerpo del feto observable por medio de los ultrasonidos. Con ayuda de la ecografía, las amniocentesis pudieron realizarse tras observar el feto por este método o durante la propia observación, para asegurarse su posición en la bolsa y evitar su daño. Mientras tanto, la pericia de la persona que realizaba la extracción pudo desarrollarse también. Desde los inicios del uso de la extracción del líquido amniótico, la pericia aumentó, generalmente asociada a cifras muy bajas de pérdida de embarazado por la extracción misma.

46 Véase Lautre, M.J. (1976), «Predicción antenatal de las anomalías comosómicas». En Botella, J., IzQuierdo, L. (eds.), Problemas actuales de genética humana, Madrid, Instituto de España, pp. 177-191.

47 Sobre la técnica ecográfica, véase Mitchell, L. M. (2001), Baby's first picture. Ultrasound and the politics of fetal subjects, Toronto, University of Toronto Press y TANSEY, E. M., Christie, D.A. (eds.)(2000), Looking at the unborn: Historical aspects of obstetric ultrasound, Londres, The Wellcome Trust, p. i, http://catalogue.wellcome.ac.uk/record=b1473079a. 
Más tarde, en la década de 1970, se introdujo el método de extracción de vellosidades del corion para muestras destinadas a análisis citogenéticos y de genética molecular. Consiste en la extracción de una pequeña muestra de vellosidad del corion (membrana fetal) justo antes de que comience su desarrollo en la placenta. La muestra se extrae por la vagina o por punción abdominal a partir de la semana octava y hasta la duodécima. Fue este tiempo, menor que el requerido para la amniocentesis, el que hizo pensar que la biopsia de corion llegaría a sustituir a la amniocentesis, pues podrían conocerse anomalías en el feto antes que por la extracción del líquido amniótico. Ambas técnicas son invasivas y llevan consigo un riesgo de pérdida del embarazo, que en el caso de la biopsia de corion fue considerado alto en los inicios de la práctica por lo que, según Schwartz-Cowan, los grupos danés y sueco que trabajaban en ello la abandonaron. Fue en un hospital de China y posteriormente en la Unión Soviética, Gran Bretaña, Francia e Italia donde los resultados de la determinación del sexo del feto mediante pruebas genéticas demostraron su éxito ${ }^{48}$.

\section{GENETIZACIÓN DEL EMBARAZO}

Se ha sugerido que el diagnóstico prenatal lleva consigo, o puede estar en la base misma, de la genetización del embarazo ${ }^{49}$. El proceso de genetización, sin embargo, atañe a la práctica médica y a la cultura biomédica pública contemporáneas, y muy intensamente desde la Segunda Guerra Mundial. Las historiografía aporta muchos datos y análisis de los cuales se extrae que la genética, el estudio sistemático de la herencia biológica por la experimentación tanto en biología como en medicina, comenzó muy pronto a estabilizar la dicotomía herencia/medio, como mundos separables a efectos de análisis clínico y descripción de enfermedades, malformaciones y desórdenes, por usar solo algunos de los términos que se tornaron usuales desde entonces. Los desarrollos de la genética molecular y los múltiples procesos por los cuales la práctica médica debía contar con bases científicas más allá del ojo clínico profesional y de las descripciones de las enfermedades infecciosas, transmisibles y heredables se encontraron, tras la Segunda Guerra Mundial, en el laboratorio y en el diseño de nuevas máquinas y aparatos de uso clínico con un creciente conocimiento de la biología molecular y

48 SCHWARTZ COWAN (2001).

49 Lippman, A. (1994), «The Genetic Construction of Prenatal Testing: Choice, Consent, or Conformity for Women?». En ROTHENBERG, THOMSON (eds.). 
celular $^{50}$. En paralelo, el desarrollo de la medicina hospitalaria en los sistemas de salud de la sociedad del bienestar puso a disposición de la ciudadanía, y de la medicina, un número permanentemente creciente de prácticas diagnósticas.

Todo lo cual se produce en plena era dorada de la investigación científica en Occidente. En España se desplegó una capacidad formativa e investigadora que, como se ha visto en los casos que se han presentado aquí, permitió adquirir conocimientos y adoptar nuevas técnicas con relativa rapidez. Las técnicas de diagnóstico prenatal y las prácticas asociadas a él, especialmente los trabajos en genética humana, comenzaron en España temprano, sujetos, puede concluirse de forma provisional, a los mismos valores culturales de genetización y tecnificación de la clínica que se extendían por otros países de la vecindad geográfica y política.

\section{CONCLUSIÓN PRELIMINAR}

Pendiente el desarrollo de una investigación más detallada sobre los primeros grupos clínicos e investigadores que introdujeron el conjunto de prácticas de diagnóstico prenatal en España, con los datos que se ofrecen pueden recuperarse algunos de los asuntos planteados en la introducción.

Como ha dicho Rayna Rapp (2000), el diagnóstico prenatal se experimenta por el propio cuerpo de las mujeres y cuando hay resultados positivos de anomalías es una experiencia personal intensa que incluye afrontar la decisión delicada de llevar adelante o no el embarazo. Las políticas en las que están envueltas estas prácticas, por su parte, incluyen derecho a la asistencia sanitaria en el embarazo, las políticas sociales, los derechos y la atención sanitaria a personas que nacen con minusvalías, entre ellas ${ }^{51}$. Esas técnicas han creado una cultura del embarazo. La investigación histórica sobre ellas sugiere que se sumaron a tendencias médicas y de investigación biológica y clínica que despegaron a finales de la década de 1950 y deben de estar en el origen del proceso de genetización del embarazo.

La práctica del diagnóstico prenatal es más que una mera técnica sanitaria aséptica pública y privada. Aparece ligada a conceptos de salud y de enfermedad que hunden sus raíces en la creciente genetización de los conceptos

50 Véase LindeE, M. Susan (2002), «Genetics disease in the 1960s: A structural revolution», American Journal of Medical Genetics 115, 75-82.

51 RAPp, R. (2000), Testing the women, testing the fetus. The social impact of amniocentesis in America, Nueva York-Londres, Routledge. 
médicos y en las prácticas experimentales. Y tiene una relación también intensa con los derechos de las propias mujeres: pese a la invisibilización del cuerpo, característica de las tecnologías médicas contemporáneas, las mujeres permanecen en el centro de las prácticas médicas y de las culturas que éstas acarrean, y el derecho al aborto por la denominada «indicación terapéutica» surge como uno de tales derechos. El diagnóstico prenatal también se ha convertido en derecho de las mujeres a conocer el estado de salud de su feto. Los procedimientos crean culturas y oferta y demanda propia de los mercados. Se trata de procedimientos que concentran la atención en los embriones y en los fetos. El concepto mismo de embarazo, medicalizado ya, se modificaba y tecnificaba, para acabar por genetizarse. Y es la introducción de esas nuevas prácticas, de esas nuevas culturas de descendencia saludable, las que he pretendido repasar de forma preliminar marcando algunos aspectos que contribuyan a comprender el proceso por el que se establecieron en España. La penalización del aborto durante toda la dictadura de Franco configuró el tipo de trabajos diagnósticos que se practicaron en embarazadas, y ese medio local y las singularidades de un estado dictatorial y oficialmente católico podrían explicar la apariencia neutra de los diagnósticos prenatales más tempranos que se hicieron en España. En ellos, la prevención de nacimientos insanos - de seres enfermos o malformados- por interrupción del embarazo no se menciona en las publicaciones médicas consultadas. La práctica de esos diagnósticos, en sus inicios en nuestro país, parece seguir simultáneamente otros códigos, relacionados con intereses profesionales clínicos y médicos, que buscaban un mayor conocimiento sobre el embarazo mismo y sobre ese sujeto tanto tiempo invisible que era el feto.

\section{AGRADECIMIENTOS}

Las investigaciones para la elaboración de este trabajo han contado con una subvención del Plan Nacional de I+D+i, Ministerio de Trabajo y Asuntos Sociales 29/03 (20031R613-CSIC) y actualmente con una del Plan Nacional de I+D+i del Ministerio de Educación y Ciencia (HUM2006-06327). Agradezco a Esther Ortega su colaboración en la detección de algunas de las fuentes citadas aquí, y a Montserrat Cabré, a Teresa Ortiz y a dos personas anónimas que lo revisaron los comentarios y sugerencias a una versión previa de este texto.

Fecha recepción: de 19 abril de 2007

Fecha aceptación: 20 de junio de 2007 